\title{
Restless legs syndrome and tension-type headache: a population-based study
}

\author{
Pil-Wook Chung ${ }^{1}$, Soo-Jin Cho ${ }^{2}$, Won-Joo Kim³ ${ }^{3}$ Kwang Ik Yang ${ }^{4}$, Chang-Ho Yun ${ }^{5}$ and Min Kyung Chu ${ }^{6 *}$
}

\begin{abstract}
Background: Recent studies have shown a significant association between restless legs syndrome (RLS) and primary headache disorders. Nevertheless, information regarding the association between tension-type headache $(T T H)$ and RLS is limited. This study aimed to investigate the association between RLS and TTH in a populationbased sample.

Methods: We selected a stratified random population sample of Koreans aged 19-69 years and assessed them using a semi-structured interview designed to identify RLS, headache type, and clinical characteristics of TTH. We determined the prevalence and clinical impact of RLS in participants with TTH.

Results: Of the 2695 participants, 570 (21.2\%) and 142 (5.3\%) were classified as having TTH and RLS, respectively. Among the 570 individuals with $T \mathrm{TH}, 113$ (19.8\%) also met the criteria for probable migraine (PM). The prevalence of RLS was significantly higher among individuals with $T$ TH than among those with non-headache $(6.0 \%$ vs $3.6 \%$, $p=0.018$ ). The prevalence of RLS was significantly higher in subjects with TTH who fulfilled PM criteria than in those with non-headache participants ( $8.0 \%$ vs. $3.6 \%, p=0.018$ ). However, RLS prevalence in individuals with TTH who did not fulfil PM criteria did not differ from that of participants with non-headache (5.5\% vs. 3.6\%, $p=0.063)$. TH participants with RLS had higher visual analogue scale scores for headache intensity (5.1 \pm 2.0 vs. $4.3 \pm 1.8, p=0.038)$, and higher prevalence of anxiety ( $20.6 \%$ vs. $8.8 \%, p=0.022)$ and depression ( $14.7 \%$ vs. $3.5 \%, p=0.002)$ than TTH participants without RLS. Multivariable analyses revealed that headache aggravation by movement (odds ratio $[\mathrm{OR}]=2.4,95 \%$ confidence interval $[\mathrm{Cl}]=1.1-5.2)$ and depression $(\mathrm{OR}=3.5,95 \% \mathrm{Cl}=1.1-11.4)$ were significant indicators of RLS among individuals with $\mathrm{TTH}$.
\end{abstract}

Conclusions: The prevalence of RLS was higher among individuals with TTH than among those with non-headache. Some clinical presentations varied in accordance with the presence of RLS among participants with $T T H$.

Keywords: Restless legs syndrome, Tension-type headache, Anxiety, Depression

\section{Background}

Tension-type headache (TTH) is the most common type of primary headache disorder and is the third most common disease in the world [1]. TTH has been considered a non-serious disorder compared to migraine. However, it is a major health problem and leads to a severe socioeconomic burden owing to its high prevalence and comorbidities such as anxiety, depression, temporo-mandibular disorders, fibromyalgia, and obstructive sleep apnoea [2-6]. Patients with

\footnotetext{
* Correspondence: chumk@hallym.ac.kr

${ }^{6}$ Department of Neurology, Kangnam Sacred Heart Hospital, Hallym

University College of Medicine, 1 Singil-ro, Yeongdeungpo-gu, Seoul 07441,

Republic of Korea

Full list of author information is available at the end of the article
}

TTH with comorbidities have more severe symptoms than those without comorbidities [2]. Therefore, identification of the associated comorbidities of TTH is important for better management of TTH and may reduce the burden of the disease.

Restless legs syndrome (RLS) is a sleep-related sensory-motor disorder characterized by unpleasant feelings in the legs, especially during rest or at bedtime. The unpleasant feelings are relieved by voluntary leg movement [7]. Previous population-based and clinic-based studies have consistently reported a significant association between RLS and migraine [8-14].

The mechanism for the association between migraine and RLS is still uncertain. Dopamine and iron 
dysregulation have been proposed to be mechanism both for migraine and RLS [7, 15-17]. Therefore, they were considered as mechanisms for the comorbidity of two disorders. Furthermore, genetic associations between RLS and migraine have been suggested [18]. Although numerous reports have suggested a close association between migraine and RLS, population-based information regarding the association between RLS and TTH, which is the most common type of headache, is limited. Nevertheless, a few studies have suggested the presence of significant associations between RLS and TTH. A population-based report indicates that RLS has a significant association with non-migraine headache [14]. A health-insurance database study in Taiwan revealed that individuals with TTH have an increased risk for developing RLS [19].

We hypothesized that RLS and TTH would have a significant association in a general population-based sample. The Korean Headache-Sleep Study (KHSS) is a cross-sectional nationwide population-based survey on sleep and headache in Korean adults aged 19-69 years and may provide an opportunity for us to assess the association between RLS and TTH. Therefore, we estimated the prevalence of TTH and RLS in a Korean general population-based sample, compared the association between TTH and RLS in comparison with individuals with non-headache, and assessed the clinical impact of RLS in individuals with TTH using data from the KHSS.

\section{Methods}

\section{Survey}

The KHSS, used in this study, was approved by the institutional review board and ethics committee of Hallym University Sacred Heart Hospital and written informed consent was obtained from all participants. Details of the KHSS have previously been described [14, 20]. Briefly, we used a 2-stage clustered random sampling method to obtain a sample proportional to the population distribution of Korea, with the exception of Jeju Island. Participants were stratified by age, sex, and occupation. We informed candidates that the survey topic was general health rather than headache and sleep to avoid interest bias. All interviewers were employees of Gallup Korea. The survey was conducted between November 2011 and January 2012 via personal visits and interviews, using a questionnaire that was designed to identify headache type, anxiety, depression, and RLS.

\section{Diagnosis of TTH}

Diagnosis of TTH was based on the criteria for infrequent TTH (code 2.1) of the third edition beta version of the International Classification of Headache Disorders (ICDH-3 beta) [21]. Participants were considered to have
TTH if they met criteria B through E (B: lasting from 30 min to 7 days; $\mathrm{C}$ : at least two of the four typical headache characteristics [i.e. bilateral pain, non-pulsating quality, mild-to-moderate pain intensity, and no aggravation by routine physical activity]; D: attacks associated with both of the following: no nausea or vomiting, not having both photophobia and phonophobia, and E: not better accounted for by another ICHD-3 diagnosis). The questionnaire used in the present study had $75.0 \%$ sensitivity and $88.2 \%$ specificity when compared to physicians' diagnoses [22]. We did not apply the frequency criterion (criterion A) in the diagnosis of TTH. Thus, patients with TTH evaluated in this study included those with infrequent TTH (code 2.1), frequent TTH (code 2.2), and chronic TTH (code 2.3). According to ICHD-3 beta, if a participant's headache met the criteria for both TTH and probable migraine (PM), he or she was considered to have TTH [21].

\section{Diagnosis of RLS}

Diagnosis of RLS was based on the Korean version paradigm of questions for the epidemiology studies of RLS $[7,23]$. The paradigm for question for the epidemiology studies for RLS was based on the International Restless Legs Syndrome Study Group criteria published in 2003 [7]. The Korean version paradigm of question for the epidemiology studies of RLS was composed of 3 questions: (1) 'Do you have unpleasant sensations in your legs combined with an urge or need to move your legs?' (2) 'Do these feelings occur mainly or only at rest and do they improve with movement?' (3) 'Are these feelings worse in the evening or night than in the morning?'. The Korean version paradigm of question for the epidemiology studies for RLS was validated with $85.3 \%$ sensitivity and $89.5 \%$ specificity by comparing doctors' diagnosis of RLS [23].

\section{Assessment of anxiety and depression}

Anxiety was measured using the Goldberg Anxiety Scale (GAS) [24]. The Korean version of GAS has $82.0 \%$ sensitivity and $94.4 \%$ specificity for the diagnosis of anxiety and has previously been validated [25]. To diagnose depression, the Patient Health Questionnaire-9 was used [26]. Participants with scores of 10 or higher on this measure were considered to have depression. The Korean version of the Patient Health Questionnaire-9 has $81.1 \%$ sensitivity and $89.9 \%$ specificity [27]. To assess headache intensity and the impact of the headache, we used the Visual Analogue Scale (VAS) and the Headache Impact Test-6, respectively.

\section{Statistical Analyses}

The Kolmogorov-Smirnov test was used to confirm the normality of the distributions. Following confirmation of 
a normal distribution, we used Student's t-tests or Analysis of Variance to compare continuous variables. In cases of non-normal distribution, we used Mann-Whitney $U$ tests or Kruskal-Wallis tests. Categorical variables were compared using Chi-square tests.

We investigated factors contributing to RLS among individuals with TTH using univariable and multivariable analyses. To perform univariable analyses, we considered factors with significant differences between TTH participants with RLS and those without RLS. To perform multivariable analyses, we developed 4 models for the association between RLS and TTH. Model 1 included sociodemographic variables (age, sex, size of residential area, and education level) and headache aggravation by movement. Model 2 added anxiety as a variable to Model 1. In Model 3, we added depression to Model 1. The final model, Model 4, incorporated sociodemographic variables, aggravation by movement, and anxiety and depression. A significance level of $p<0.05$ was used for all analyses. Analyses were conducted using the Statistical Package for the Social Sciences 22.0 (SPSS 22.0; IBM, Armonk, NY).

Similar to most survey sampling designs, there were missing data (resulting from non-response) for several variables. All of the reported results are based on the available data. As such, the total numbers for some variables diverge from 2695 because of missing data for that particular variable. Imputation techniques were not used because we wanted to minimize non-response effects [28].

\section{Results}

\section{Survey}

Interviewers approached 7430 individuals. Of the 7430 individuals, 3114 agreed to participate in this survey (rejection rate, 58.1\%). Of these participants, 2695 completed the survey (cooperation rate, 36.3\%; Fig. 1). Distributions of age, sex, size of residential area, and education level were not significantly different between our survey participants and the general population of Korea (Additional file 1: Table S1).

\section{Prevalence of TTH and RLS}

Of the 2695 participants, 570 (21.2\%) were considered to have TTH and 1422 (52.8\%) were classified as nonheadache participants. Of the 570 participants who were classified as TTH, 14 participants $(2.5 \%)$ were classified as chronic TTH (code 2.3), $229(40.2 \%)$ as infrequent episodic TTH (code 2.1), and $327(57.3 \%$ ) as frequent episodic TTH (code 2.2). Among the individuals with TTH, $113(19.8 \%)$ also met the criteria for PM. The prevalence of TTH was not significantly affected by age, sex, or educational level. The prevalence of TTH was higher in rural areas compared to large cities and small-to-medium cities (Table 1). One hundred

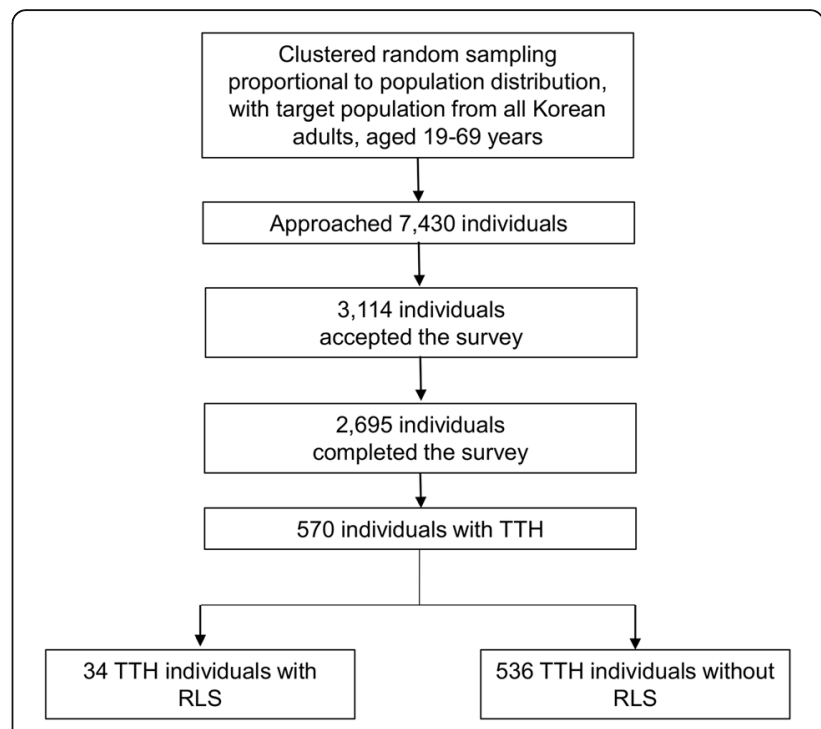

Fig. 1 Flowchart depicting the participation of subjects in the Korean Headache-Sleep Study. RLS, restless legs syndrome; TTH, tension-type headache

and forty-two (5.3\%) participants were classified as having RLS. Restless legs syndrome was more prevalent in women and participants with lower educational level (middle school or less) than among men and those with higher educational level. The prevalence of RLS had a trend toward an increase with increasing age (Additional file 1: Table S1). Sociodemographic characteristics of individuals with TTH and non-headache are summarized in Table 1. Women and participants living in rural areas were more common among individuals with TTH than among those with non-headache. However, distributions of age and educational level were not significantly different between the two groups.

\section{Prevalence of anxiety and depression}

Two-hundred and sixty-eight (9.9\%) and 116 (4.3\%) participants were classified as having anxiety and depression, respectively. The prevalence of anxiety $(9.5 \%$ vs. $5.3 \%, p=0.001)$ and depression $(4.2 \%$ vs. $1.8 \%, p=0.001)$ were significantly higher among individuals with TTH than among those with non-headache.

\section{Prevalence of RLS in participants with TTH}

Among the 570 individuals with TTH, 34 (6.0\%) were classified as having RLS. The prevalence of RLS was significantly higher among individuals with TTH than among individuals with non-headache $(6.0 \%$ vs $3.6 \%$, $p=0.018$ ). Among the 113 participants with TTH who fulfilled PM criteria, 9 participants $(8.0 \%)$ were classified as having RLS. The prevalence of RLS among participants with TTH who also fulfilled PM 
Table 1 Sociodemographic characteristics and RLS status in participants with TTH and participants with non-headache

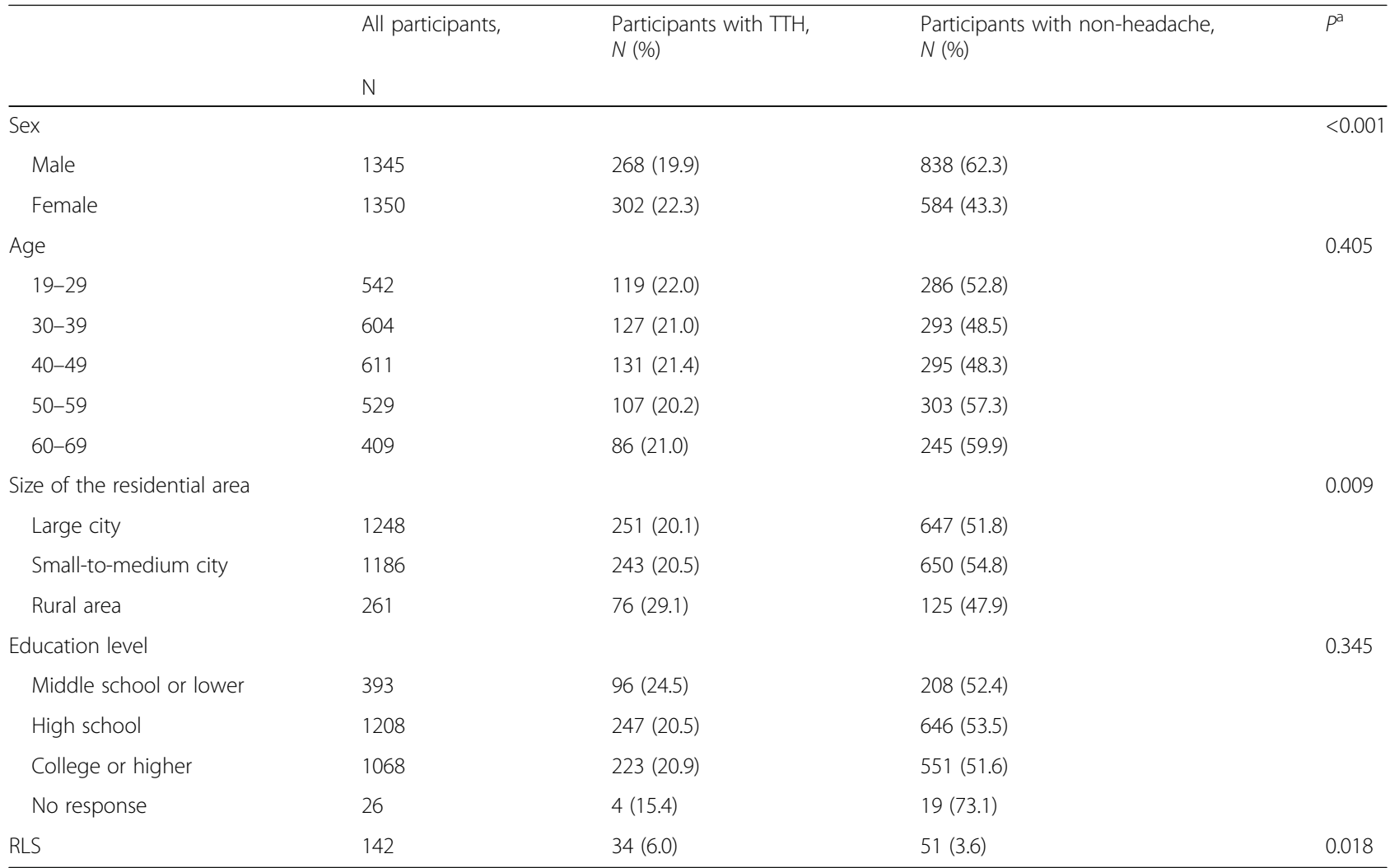

${ }^{a}$ Comparison between participants with tension-type headache and those without headache. $T H H$ Tension-type headache, $R L S$ Restless legs syndrome

criteria was not significantly different from that among those with TTH not fulfilling PM criteria $(8.0 \%$ vs. $5.5 \%, p=0.318)$. The prevalence of RLS in participants with TTH not fulfilling PM criteria did not differ from that of individuals with non-headache (5.5\% vs. $3.6 \%, p=0.063)$. Nevertheless, the prevalence of RLS among individuals with TTH fulfilling PM criteria was significantly higher than that among those with non-headache (8.0\% vs. 3.6\%, $p=0.018$; Fig. 2). Prevalence of RLS was analysed according to headache frequency. Prevalence of RLS in participants with TTH with 1-10 attacks per month $(5.9 \%, p=0.861)$ and participants with TTH with $>10$ attacks per month $(13.6 \%$, $p=0.116)$ was numerically higher compared to that of those with $<1$ attack per month (5.5\%). However, it did not reach statistical significance.

We also investigated the prevalence of RLS in individuals with TTH grouped on the basis of anxiety and depression status (Table 2). The prevalence of RLS was significantly higher among TTH participants with anxiety than among those without anxiety $(13.0 \%$ vs. $5.2 \%, p=0.022)$. The prevalence of RLS was significantly higher among TTH subjects with depression than among those without depression $(20.8 \%$ vs. $5.3 \%, p=0.002)$.

\section{Demographics and clinical presentations of participants with TTH according to RLS status}

The demographics and clinical presentations of participants with TTH according to the presence of RLS are summarized in Table 2. Demographic variables, headache characteristics (except for non-aggravation by

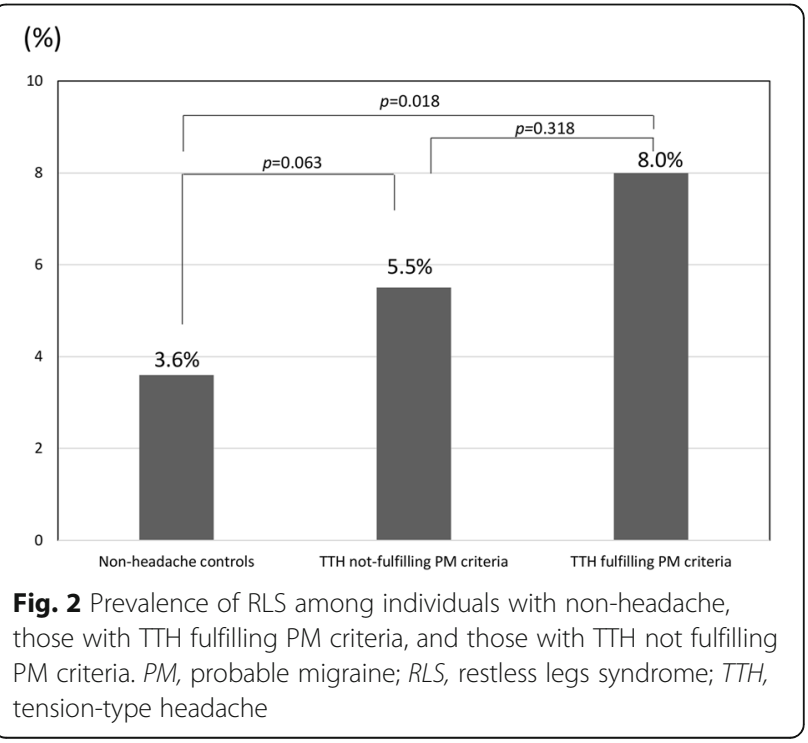


Table 2 Demographics, headache characteristics, and associated symptoms in subjects with $\mathrm{TTH}$ according to the presence of RLS

\begin{tabular}{|c|c|c|c|}
\hline & $\begin{array}{l}\text { TTH } \\
\text { subjects } \\
\text { with RLS }\end{array}$ & $\begin{array}{l}\text { TTH } \\
\text { subjects } \\
\text { without } \\
\text { RLS }\end{array}$ & $P$ \\
\hline & $N=34$ & $N=536$ & \\
\hline \multicolumn{4}{|l|}{ Demographics } \\
\hline Mean age $\pm S D$ (years) & $46.4 \pm 13.3$ & $42.5 \pm 13.7$ & 0.108 \\
\hline Women, N (\%) & $20(58.8)$ & $282(52.6)$ & 0.482 \\
\hline \multicolumn{4}{|l|}{ Headache characteristics } \\
\hline Bilateral pain, $N(\%)$ & $23(67.6)$ & $351(65.5)$ & 0.797 \\
\hline $\begin{array}{l}\text { Non-pulsating quality, } \\
N(\%)\end{array}$ & $16(47.1)$ & $211(39.4)$ & 0.374 \\
\hline $\begin{array}{l}\text { Mild-to-moderate severity, } \\
N(\%)\end{array}$ & $34(100.0)$ & $526(98.1)$ & 0.422 \\
\hline $\begin{array}{l}\text { Non-aggravation by movement, } \\
N(\%)\end{array}$ & $22(64.7)$ & $429(80.0)$ & 0.033 \\
\hline \multicolumn{4}{|l|}{ Associated symptoms } \\
\hline Photophobia, N (\%) & $5(14.7)$ & $41(7.6)$ & 0.143 \\
\hline Phonophobia, N (\%) & $9(26.5)$ & $172(32.1)$ & 0.495 \\
\hline Osmophobia, N (\%) & $7(20.6)$ & $88(16.4)$ & 0.527 \\
\hline \multicolumn{4}{|l|}{ Mood } \\
\hline Anxiety & $7(20.6)$ & $47(8.8)$ & 0.022 \\
\hline Depression & $5(14.7)$ & $19(3.5)$ & 0.002 \\
\hline Headache frequency per month & $2.3 \pm 3.5$ & $1.9 \pm 4.9$ & 0.054 \\
\hline VAS score for headache intensity & $5.1 \pm 2.0$ & $4.3 \pm 1.8$ & 0.038 \\
\hline
\end{tabular}

RLS Restless legs syndrome, $T H$ Tension-type headache, VAS Visual Analogue Scale

movement), and accompanying symptoms were not significantly different between TTH subjects with RLS and those without RLS. Visual Analogue Scale scores for headache intensity were significantly higher among TTH subjects with RLS than among those without RLS. Headache frequency was marginally different between the two groups, although this difference was not significant. The prevalence of anxiety and depression were significantly higher among participants with TTH with RLS than in those without RLS.

\section{Factors contributing to RLS among participant with TTH}

Univariate analyses revealed that headache aggravation by movement (odds ratio $[\mathrm{OR}]=2.2,95 \%$ confidence interval $[\mathrm{CI}]=1.1-4.6)$, anxiety $(\mathrm{OR}=2.7,95 \% \mathrm{CI}=1.2-6.5)$, and depression $(\mathrm{OR}=4.7,95 \% \mathrm{CI}=1.6-13.5)$ are associated with an increased risk for RLS. Multivariable analyses including sociodemographic variables and aggravation by movement (Model 1) indicated that aggravation by movement leads to an increased OR for RLS (OR $=2.3,95 \%$ $\mathrm{CI}=1.1-4.8$ ). In Model 2 (including sociodemographic variables, aggravation by movement, and anxiety), aggravation by movement $(\mathrm{OR}=2.3,95 \% \mathrm{CI}=1.1-4.9)$ and anxiety $(\mathrm{OR}=3.4,95 \% \mathrm{CI}=1.3-8.8)$ had significant effects on RLS status. In Model 3 (including sociodemographic variables, aggravation by movement, and depression), aggravation by movement ( $\mathrm{OR}=2.4,95 \%$ $\mathrm{CI}=1.1-5.2)$ and depression $(\mathrm{OR}=4.9,95 \% \mathrm{CI}=1.6-15.0)$ led to increased ORs for RLS. Model 4 (including sociodemographic variables, aggravation by movement, anxiety, and depression) revealed that aggravation by movement $(\mathrm{OR}=2.4,95 \% \mathrm{CI}=1.1-5.2)$ and depression $(\mathrm{OR}=3.5$, $95 \% \mathrm{CI}=1.1-11.4$ ) have significant effects on RLS status (Table 3).

\section{Discussion}

The main findings of the present study are as follows: 1 ) The prevalence of TTH and RLS are $21.1 \%$ and 5.3\%, respectively, in the Korean general population sample used; 2) The prevalence of RLS among subjects with TTH was significantly higher than among participants with non-headache; and 3) TTH participants with RLS had more severe headache intensity and higher prevalence of anxiety and depression than those without RLS.

While a significant association between RLS and migraine has been consistently reported, information regarding the association between RLS and TTH, which is another common primary headache, is limited. Recently, a retrospective cohort study using a health insurance database suggested that patients with TTH had an increased risk of developing RLS [19]. Nevertheless, the study did not provide cross-sectional evidence for an association between RLS and TTH, identified TTH and RLS cases on the basis of diagnostic codes rather than diagnostic criteria and did not assess the impact of RLS on the clinical presentation of TTH. Here, we identified RLS and TTH cases on the basis of validated criteria and found that RLS was more prevalent among individuals with TTH than among those with non-headache in a general population-based sample. In addition, we found that participants with TTH with RLS had more severe headache intensity than those with TTH without RLS.

In the present study, TTH individuals with anxiety and depression had higher RLS prevalence than those without anxiety and depression. Multivariable regression analyses revealed that depression is an independent predictor of RLS. These findings are concurrent with those of previous studies of the association between RLS and mood symptoms. Individuals with anxiety or depression have been shown to have a higher risk of RLS and vice versa [29-31]. Anxiety and depression are also common comorbid conditions of TTH [3]. Therefore, it is plausible that mood symptoms, such as anxiety and depression, are important factors linking RLS to TTH. Further studies are required among $\mathrm{TTH}$ subjects regarding 
Table 3 Univariate and multivariable logistic regression analyses of RLS among individuals with tension-type headache ( $N=570)$

\begin{tabular}{|c|c|c|c|c|c|}
\hline & \multirow[t]{2}{*}{ Univariable analyses } & \multicolumn{4}{|c|}{ Multivariable analyses } \\
\hline & & Model $1^{a}$ & Model $2^{b}$ & Model $3^{c}$ & Model $4^{d}$ \\
\hline & OR $(95 \% \mathrm{Cl})$ & OR $(95 \% \mathrm{Cl})$ & OR $(95 \% \mathrm{Cl})$ & OR $(95 \% \mathrm{Cl})$ & OR $(95 \% \mathrm{Cl})$ \\
\hline Aggravation by movement & $2.2(1.1-4.6)$ & $2.3(1.1-4.8)$ & $2.3(1.1-4.9)$ & $2.4(1.1-5.2)$ & $2.4(1.1-5.2)$ \\
\hline Anxiety & $2.7(1.2-6.5)$ & & $3.4(1.3-8.8)$ & & $2.6(0.9-7.0)$ \\
\hline Depression & $4.7(1.6-13.5)$ & & & $4.9(1.6-15.0)$ & $3.5(1.1-11.4)$ \\
\hline
\end{tabular}

$O R$ odds ratio, $\mathrm{Cl}$ confidence interval

ancluding sociodemographic variables (age, sex, size of residential area, and educational level) and aggravation by movement

Including sociodemographic variables, aggravation by movement, and anxiety

'Including sociodemographic variables, aggravation by movement, and depression

${ }^{d}$ Including sociodemographic variables, aggravation by movement, anxiety, and depression

mood symptoms and RLS to elucidate the associations among RLS, mood symptoms, and TTH.

We classified 113 participants fulfilling both TTH and PM criteria as subjects with TTH in accordance with the general rule of ICHD-3 beta. Although RLS prevalence in participants with TTH fulfilling PM criteria did not significantly differ from that in those with TTH not fulfilling PM criteria, RLS prevalence in participants with TTH fulfilling PM criteria was significantly higher than in those with non-headache. However, RLS prevalence among participants with TTH not fulfilling PM criteria was not different from that in participants with nonheadache (Fig. 2). Among migrainous features, aggravation by movement was more frequently found in TTH participants with RLS than in those without RLS (Table 2). Univariable and multivariable analyses indicated that headache aggravation by movement is an independent predictor of RLS among participants with TTH (Table 3). These findings were concurrent with previous findings that the frequency of RLS may increase with an increase in the number of migrainous symptoms among migraineurs [8]. However, other migrainous features, such as unilateral pain, pulsating quality, moderate-to-severe headache intensity, photophobia, and phonophobia were not significantly different between individuals with and those without RLS in the present study. Further studies including various headache populations will validate the association between migrainous features and RLS among headache sufferers.

The 1-year TTH prevalence rate $(21.2 \%)$ in the present study was somewhat lower than that previously observed in Western countries (20-78\%) [32]. The 1-year prevalence of TTH in Asian countries ranges from 10.3 to $33.3 \%$ in most studies of Asian populations, which is somewhat lower than those reported in studies of European and North American countries [32, 33]. RLS prevalence $(5.3 \%)$ in the present study was similar to those reported in previous studies from Korea and other Asian countries, which range from 1.8 to $8.3 \%$. Similar to TTH, the reported RLS prevalence in Asian populations is lower than that observed in Western countries [5, 23, 30, 34]. Therefore, the prevalence of RLS and TTH in the present study is similar to the reported prevalence in Asian countries. The similarities between the prevalence of RLS and TTH in the present study and in previous reports from Asian countries suggest that our study properly evaluated RLS and TTH.

Restless leg syndrome has long been considered to be caused by dopaminergic system dysfunction and disturbed iron metabolism [16, 35]. Unbalanced level of dopaminergic neurotransmitter might also be involved in migraine pathogenesis and dysfunctional iron metabolism in the brain of migraine has been suggested $[15,17,18]$. Therefore, it is plausible that some underlying migraine characteristics may be a substrate for linking TTH and RLS in consideration of more strong association between TTH fulfilling PM criteria and RLS than between TTH not fulfilling PM criteria and RLS in the present study.

In the present study, depression was significant contributing factor for RLS among individuals with TTH in univariable and multivariable analyses. Association of depression and RLS has been documented in populationbased studies [34, 36]. Dopaminergic dysfunction was noted in depression [37]. It also has been considered as a key mechanism of RLS as mentioned above [35]. Dopaminergic dysregulation in TTH has been proposed in previous studies [38, 39]. Accordingly, dopaminergic dysfunction could be a mechanism for connecting depression and RLS among individuals with TTH. Further experimental and clinical studies were needed for the association of depression, RLS and TTH.

There are several limitations to the present study. Firstly, we diagnosed RLS by using the Korean version paradigm of questions for the epidemiology studies of RLS based on participant's report [23]. Therefore, some conditions similar to RLS may have been included. Akathisia, meralgia paresthetica, peripheral neuropathy, and nocturnal cramping can mimic RLS. Nevertheless, the Korean version paradigm of questions for the epidemiology studies of RLS showed high sensitivity and 
specificity for the diagnosis of RLS when comparing doctors' diagnosis of RLS [7, 23]. Secondly, we did not assess the severity of RLS owing to limitations on questionnaire length. An attempt to quantitatively correlate RLS and headache may add more insight to the association between TTH and RLS. Thirdly, although the current study used a population-based sample with low sampling error, its statistical power was limited in terms of examining the subgroups of interest. In other words, the lack of significant findings in the subgroup analyses might be the result of the limited sample size.

Our study has several strengths. Firstly, we used the data of KHSS which was based on clustered random sampling proportional to the Korean population distribution with low sampling error. This condition allowed us to precisely investigate the association between RLS and TTH in a population-base setting. Secondly, we investigated anxiety and depression, which are common comorbidities among individuals of RLS and assessed the effect of anxiety and depression in the association between RLS and TTH. Thirdly, we assessed the prevalence of RLS among individuals with TTH according to migrainous features.

\section{Conclusions}

In conclusion, the prevalence of RLS among individuals with TTH was significantly higher than that among those with non-headache in a population-based sample in Korea. TTH participants with RLS had higher VAS scores for headache intensity than those without RLS. Headache aggravation by movement and depression were significant indicators of the presence of RLS among individuals with TTH. Our findings may provide a better understanding of the comorbidity between RLS and TTH.

\section{Additional file}

Additional file 1: Table S1. Sociodemographic characteristics of survey participants, the total Korean population, and cases identified as having TTH and RLS. (DOCX $20 \mathrm{~kb}$ )

\begin{abstract}
Abbreviations
Cl: Confidence interval; GAS: Goldberg anxiety scale; ICHD: International classification of headache disorders; IRLSSG: International restless legs syndrome study group; KHSS: Korean headache-sleep study; OR: Odds ratio; PM: Probable migraine; RLS: Restless legs syndrome; TTH: Tension-type headache; VAS: Visual analogue scale
\end{abstract}

\section{Acknowledgements}

The authors would like to thank Gallup Korea for providing technical support for the Korean Headache-Sleep study.

\section{Funding}

This study was supported by a 2011 grant from the Korean Academy of Medical Sciences.

\section{Author's contributions}

MKC conceived and designed the study, collected and analyzed data, and wrote the manuscript. PWC conceived and designed the study, and wrote the manuscript. SJC designed the study and analyzed data. WJK conceived the study and collected the data. KIY conceived the study and analyzed data. $\mathrm{CHY}$ conceived and designed the study, and collected data. All authors read and approved the final manuscript.

\section{Competing interests}

Dr. Pil-Wook Chung declared no conflict of interest.

Dr. Soo-Jin Cho was involved as a site investigator of multicenter trial sponsored by Otsuka Korea, Eli Lilly and Company, Korea BMS, and Eisai Korea, and received research support from Hallym University Research Fund 2016 and received lecture honoraria from Yuyu Pharmaceutical Company. Dr. Won-Joo Kim declared no conflict of interest.

Dr. Kwang Ik Yang declared no conflict of interest.

Dr. Chang-Ho Yun declared no conflict of interest.

Dr. Min Kyung Chu was as a site investigator for a multicenter trial sponsored by Eli Lilly, worked an advisory member for Teva, and received lecture honoraria from Allergan Korea and Yuyu Pharmaceutical Company.

\section{Publisher's Note}

Springer Nature remains neutral with regard to jurisdictional claims in published maps and institutional affiliations.

\section{Author details}

${ }^{1}$ Department of Neurology, Kangbuk Samsung Hospital, Sungkyunkwan University School of Medicine, Seoul, Republic of Korea. ${ }^{2}$ Department of Neurology, Dongtan Sacred Heart Hospital, Hallym University College of Medicine, Hwaseong, Republic of Korea. ${ }^{3}$ Department of Neurology, Gangnam Severance Hospital, Yonsei University, College of Medicine, Seoul, Republic of Korea. ${ }^{4}$ Department of Neurology, Soonchunhyang University College of Medicine, Cheonan Hospital, Cheonan, Republic of Korea.

${ }^{5}$ Department of Neurology, Bundang Clinical Neuroscience Institute, Seoul National University Bundang Hospital, Seongnam, Republic of Korea. ${ }^{6}$ Department of Neurology, Kangnam Sacred Heart Hospital, Hallym University College of Medicine, 1 Singil-ro, Yeongdeungpo-gu, Seoul 07441, Republic of Korea.

Received: 7 March 2017 Accepted: 8 March 2017

Published online: 19 April 2017

\section{References}

1. Global Burden of Disease Study 2013 Collaborators (2015) Global, regional, and national incidence, prevalence, and years lived with disability for 301 acute and chronic diseases and injuries in 188 countries, 1990-2013: a systematic analysis for the Global Burden of Disease Study 2013. Lancet 386(9995):743-800. doi:10.1016/50140-6736(15)60692-4

2. Puca F, Genco S, Prudenzano MP, Savarese M, Bussone G, D'Amico D et al (1999) Psychiatric comorbidity and psychosocial stress in patients with tension-type headache from headache centers in Italy. The Italian collaborative group for the study of psychopathological factors in primary headaches. Cephalalgia 19(3):159-164

3. Song TJ, Cho SJ, Kim WJ, Yang KI, Yun CH, Chu MK (2016) Anxiety and depression in tension-type headache: a population-based study. PLoS One 11(10):e0165316. doi:10.1371/journal.pone.0165316

4. Freedom T, Evans RW (2013) Headache and sleep. Headache 53(8): 1358-1366. doi:10.1111/head.12178

5. Ohayon MM, O'Hara R, Vitiello MV (2012) Epidemiology of restless legs syndrome: a synthesis of the literature. Sleep Med Rev 16(4):283-295

6. Cho YW, Shin WC, Yun CH, Hong SB, Kim JH, Allen RP, Earley CJ (2008) Epidemiology of restless legs syndrome in Korean adults. Sleep 31(2):219-223

7. Allen RP, Picchietti D, Hening WA, Trenkwalder C, Walters AS, Montplaisi J (2003) Restless legs syndrome: diagnostic criteria, special considerations, and epidemiology. A report from the restless legs syndrome diagnosis and epidemiology workshop at the National Institutes of Health. Sleep Med 4(2): 101-119

8. Chen PK, Fuh JL, Chen SP, Wang SJ (2010) Association between restless legs syndrome and migraine. J Neurol Neurosurg Psychiatry 81(5):524-528. doi: 10.1136/jnnp.2009.191684 
9. Suzuki S, Suzuki K, Miyamoto M, Miyamoto T, Watanabe $Y$, Takashima R et al (2011) Evaluation of contributing factors to restless legs syndrome in migraine patients. J Neurol 258(11):2026-2035. doi:10.1007/s00415-011-6064-3

10. Winter AC, Schurks M, Berger K, Buring JE, Gaziano JM, Kurth T (2013) Migraine and restless legs syndrome in men. Cephalalgia 33(2):130-135. doi: $10.1177 / 0333102412466965$

11. Zanigni S, Giannini G, Melotti R, Pattaro C, Provini F, Cevoli S et al (2014) Association between restless legs syndrome and migraine: a populationbased study. Eur J Neurol 21(9):1205-1210. doi:10.1111/ene.12462

12. Schurks M, Winter A, Berger K, Kurth T (2014) Migraine and restless legs syndrome: a systematic review. Cephalalgia 34(10):777-794. doi:10.1177/ 0333102414537725

13. Yang FC, Lin TY, Chen HJ, Lee JT, Lin CC, Huang WY et al (2016) Increased risk of restless legs syndrome in patients with migraine: a nationwide population-based cohort study. Medicine (Baltimore) 95(5):e2646. doi:10. 1097/md.0000000000002646

14. Cho SJ, Chung YK, Kim JM, Chu MK (2015) Migraine and restless legs syndrome are associated in adults under age fifty but not in adults over fifty: a population-based study. J Headache Pain 16:554. doi:10.1186/s10194015-0554-0

15. D'Andrea G, D'Arrigo A, Dalle Carbonare M, Leon A (2012) Pathogenesis of migraine: role of neuromodulators. Headache 52(7):1155-1163. doi:10.1111/j. 1526-4610.2012.02168.x

16. Earley CJ, Connor J, Garcia-Borreguero D, Jenner P, Winkelman J, Zee PC et al (2014) Altered brain iron homeostasis and dopaminergic function in restless legs syndrome (Willis-Ekbom disease). Sleep Med 15(11):1288-1301. doi:10.1016/j.sleep.2014.05.009

17. Kruit MC, Launer LJ, Overbosch J, van Buchem MA, Ferrari MD (2009) Iron accumulation in deep brain nuclei in migraine: a population-based magnetic resonance imaging study. Cephalalgia 29(3):351-359. doi:10.1111/ j.1468-2982.2008.01723.x

18. Fuh JL, Chung MY, Yao SC, Chen PK, Liao YC, Hsu CL et al (2015) Susceptible genes of restless legs syndrome in migraine. Cephalalgia. doi:10. 1177/0333102415620907

19. Yang FC, Lin TY, Chen HJ, Lee JT, Lin CC, Kao CH (2015) Risk of restless legs syndrome following tension-type headache: a nationwide population-based cohort study. Medicine (Baltimore) 94(46):e2109. doi:10.1097/md. 0000000000002109

20. Oh K, Cho SJ, Chung YK, Kim JM, Chu MK (2014) Combination of anxiety and depression is associated with an increased headache frequency in migraineurs: a population-based study. BMC Neurol 14:238. doi:10.1186/ s12883-014-0238-4

21. Headache Classification Committee of the International Headache Society (IHS) The International Classification of Headache Disorders, 3rd edition (beta version) (2013). Cephalalgia 33 (9):629-808. doi:10.1177/ 0333102413485658

22. Kim BK, Chu MK, Lee TG, Kim JM, Chung CS, Lee KS (2012) Prevalence and impact of migraine and tension-type headache in Korea. J Clin Neurol 8(3) 204-211. doi:10.3988/jen.2012.8.3.204

23. Cho YW, Lee MY, Yun CH, Shin WC, Hong SB, Kim JH (2007) The reliability and validity of the Korean version of paradigm of questions for epidemiology studies of restless legs syndrome and the Johns Hopkins telephone diagnostic interview form for the restless legs syndrome. J Kor Nerol Assoc 25(4):494-499

24. Goldberg D, Bridges K, Duncan-Jones P, Grayson D (1988) Detecting anxiety and depression in general medical settings. BMJ 297(6653):897-899

25. Park HS, Lim JY, Lee SH, Cha YS, Sun WS (2001) Reliability and validity of anxiety screening scale. Korean J Fam Med 22(8):1224-1232

26. Pignone MP, Gaynes BN, Rushton JL, Burchell CM, Orleans CT, Mulrow CD et al (2002) Screening for depression in adults: a summary of the evidence for the U.S. Preventive Services Task Force. Ann Intern Med 136(10):765-776

27. Choi HS, Choi JH, Park KH, Joo KJ, Ga H, Ko HJ et al (2007) Standardization of the Korean version of patient health questionnaire- 9 as a screening instrument for major depressive disorder. Korean J Fam Med 28(2):114-119

28. Rubin DB, Little RJ (2002) Statistical analysis with missing data. J Wiley \& Sons, Hoboken

29. Sevim S, Dogu O, Kaleagasi H, Aral M, Metin O, Camdeviren H (2004) Correlation of anxiety and depression symptoms in patients with restless legs syndrome: a population based survey. J Neurol Neurosurg Psychiatry 75(2):226-230
30. Nomura T, Inoue Y, Kusumi M, Uemura Y, Nakashima K (2008) Prevalence of restless legs syndrome in a rural community in Japan. Mov Disord 23(16): 2363-2369. doi:10.1002/mds.22274

31. Becker PM, Sharon D (2014) Mood disorders in restless legs syndrome (Willis-Ekbom disease). J Clin Psychiatry 75(7):e679-694. doi:10.4088/JCP. $13 \mathrm{r} 08692$

32. Stovner L, Hagen K, Jensen R, Katsarava Z, Lipton R, Scher A et al (2007) The global burden of headache: a documentation of headache prevalence and disability worldwide. Cephalalgia 27(3):193-210

33. Peng KP, Wang SJ (2014) Epidemiology of headache disorders in the AsiaPacific region. Headache 54(4):610-618

34. Kim WH, Kim BS, Kim SK, Chang SM, Lee DW, Cho MJ et al (2012) Restless legs syndrome in older people: a community-based study on its prevalence and association with major depressive disorder in older Korean adults. Int J Geriatr Psychiatry 27(6):565-572

35. Allen R (2004) Dopamine and iron in the pathophysiology of restless legs syndrome (RLS). Sleep Med 5(4):385-391. doi:10.1016/j.sleep.2004.01.012

36. Lee HB, Hening WA, Allen RP, Kalaydjian AE, Earley CJ, Eaton WW et al (2008) Restless legs syndrome is associated with DSM-IV major depressive disorder and panic disorder in the community. J Neuropsychiatry Clin Neurosci 20(1):101-105

37. Dunlop BW, Nemeroff CB (2007) The role of dopamine in the pathophysiology of depression. rch. Gen Psychiatry 64(3):327-337

38. Castillo J, Martinez F, Leira R, Lema M, Noya M (1994) Plasma monoamines in tension-type headache. Headache 34(9):531-535

39. Friedman BW, Adewunmi V, Campbell C, Solorzano C, Esses D, Bijur PE et al (2013) A randomized trial of intravenous ketorolac versus intravenous metoclopramide plus diphenhydramine for tension-type and all nonmigraine, noncluster recurrent headaches. Annal Emerg Med 62(4):311-318, e314

\section{Submit your manuscript to a SpringerOpen ${ }^{\circ}$ journal and benefit from:}

- Convenient online submission

- Rigorous peer review

- Immediate publication on acceptance

- Open access: articles freely available online

- High visibility within the field

- Retaining the copyright to your article

Submit your next manuscript at $>$ springeropen.com 